\title{
Evaluation and comparison of bioinformatic tools for the enrichment analysis of metabolomics data
}

\author{
Anna Marco-Ramell ${ }^{1,2}$, Magali Palau-Rodriguez ${ }^{1,2}$, Ania Alay ${ }^{3}$, Sara Tulipani ${ }^{1}$, Mireia Urpi-Sarda ${ }^{1,2}$, \\ Alex Sanchez-Pla ${ }^{3,4}$ and Cristina Andres-Lacueva ${ }^{1,2^{*}}$
}

\begin{abstract}
Background: Bioinformatic tools for the enrichment of 'omics' datasets facilitate interpretation and understanding of data. To date few are suitable for metabolomics datasets. The main objective of this work is to give a critical overview, for the first time, of the performance of these tools. To that aim, datasets from metabolomic repositories were selected and enriched data were created. Both types of data were analysed with these tools and outputs were thoroughly examined.
\end{abstract}

Results: An exploratory multivariate analysis of the most used tools for the enrichment of metabolite sets, based on a non-metric multidimensional scaling (NMDS) of Jaccard's distances, was performed and mirrored their diversity. Codes (identifiers) of the metabolites of the datasets were searched in different metabolite databases (HMDB, KEGG, PubChem, ChEBI, BioCyc/HumanCyc, LipidMAPS, ChemSpider, METLIN and Recon2). The databases that presented more identifiers of the metabolites of the dataset were PubChem, followed by METLIN and ChEBI. However, these databases had duplicated entries and might present false positives. The performance of over-representation analysis (ORA) tools, including BioCyc/HumanCyc, ConsensusPathDB, IMPaLA, MBRole, MetaboAnalyst, Metabox, MetExplore, MPEA, PathVisio and Reactome and the mapping tool KEGGREST, was examined. Results were mostly consistent among tools and between real and enriched data despite the variability of the tools. Nevertheless, a few controversial results such as differences in the total number of metabolites were also found. Disease-based enrichment analyses were also assessed, but they were not found to be accurate probably due to the fact that metabolite disease sets are not up-to-date and the difficulty of predicting diseases from a list of metabolites.

Conclusions: We have extensively reviewed the state-of-the-art of the available range of tools for metabolomic datasets, the completeness of metabolite databases, the performance of ORA methods and disease-based analyses. Despite the variability of the tools, they provided consistent results independent of their analytic approach. However, more work on the completeness of metabolite and pathway databases is required, which strongly affects the accuracy of enrichment analyses. Improvements will be translated into more accurate and global insights of the metabolome.

Keywords: Bioinformatic tools, Database, Enrichment, HumanCyc, KEGG, Metabolite, Metabolomics, Over-representation analysis, Pathway, Reactome

\footnotetext{
* Correspondence: candres@ub.edu

'Biomarkers \& Nutrimetabolomics Laboratory, Nutrition, Food Science and

Gastronomy Department, Food Technology Reference Net (XaRTA), Nutrition and Food Safety Research Institute (INSA-UB), Faculty of Pharmacy and Food Sciences, Pharmacy and Food Science Faculty, University of Barcelona,

Barcelona, Spain

${ }^{2}$ CIBER Fragilidad y Envejecimiento Saludable [CIBERfes], Instituto de Salud

Carlos III [ISCIII], Madrid, Spain

Full list of author information is available at the end of the article
} 


\section{Background}

Enrichment techniques for 'omics' data are key tools for understanding complex biological systems. These tools reduce the complexity of the data, improve interpretation and understanding of biological systems, and help to generate hypotheses. Although the number of tools for 'omics' is rapidly growing, suitable tools for metabolomics are still scarce. Most of the available tools for metabolomics data have been previously developed for other 'omics' technologies. These tools have been described in detail elsewhere [1-6].

Enrichment tools denote any analytic technique that benefits from molecular pathway or network information to gain insight into a biological system [4]. The most widely used methodology for performing such analysis is termed functional enrichment or over-representation analysis (ORA) [7]. This analysis looks for keywords or descriptors of the set of molecules of interest (e.g. those over-expressed) with respect to a background reference set (e.g. the whole genome/transcriptome/proteome/metabolome or the set of molecules detected by the technology employed) [1]. Classical enrichment analyses employ Fisher's exact test, but many other enrichment methods have derived from it, e.g. hypergeometric, KolmogorovSmirnov or Wilcoxon statistical tests $[6,7]$.

To the best of our knowledge studies evaluating the performance of enrichment tools for metabolite sets do not exist yet. The aim of the present work will be to dissect, for the first time, these techniques. First of all, we have carried out an exploratory multivariate analysis of the state-of-theart of bioinformatic tools for metabolomics sets to visualize their diversity. Then, we have examined the completeness of metabolite databases, the performance of ORA methods and accuracy of disease-based analyses. For these purposes, we have used datasets from metabolomic repositories, whose results have been already published in peerreviewed journals. In addition, we have enriched selected metabolic pathways and then compared the outputs of these tools when using real datasets or enriched data. Thus the present study provides a global insight of the current status of bioinformatic tools for the analysis and interpretation of metabolite sets from metabolomic studies.

\section{Methods}

\section{Datasets}

The list of metabolites used in this work refers to five datasets from metabolomics studies in humans, already published in peer-reviewed journals, whose raw data, study information and the list of identified metabolites are available in MetabolomeXchange [8], an online portal of metabolomics repositories including MetaboLights [9], Metabolomics Workbench [10] and Metabolomic Repository Bordeaux. A brief summary of the datasets is shown in Table 1. These datasets correspond to the following publications: 1) Lanza et al. [11]; 2) Fiehn et al. [12]; 3) Kaluarachchi et al. [13]; 4) Hart et al. [14]; and 5) Zhu et al. [15]. P- and adjusted $p$-values were obtained from the original papers [11-15] and only metabolites with an adjusted $p$-value $<0.05$ were used for tools comparison. The list of metabolites is shown as (Additional file 1: Table S1).

\section{Search of metabolite identifiers}

Bioinformatic tools for enrichment analysis require the metabolite name or code (identifier) from a metabolite database. Although Kyoto Encyclopaedia of Genes and Genomes (KEGG Compound) identifiers [16] are the most commonly used in metabolomics [3, 17], some tools prefer other database identifiers such as PubChem [18], BioCyc/HumanCyc (hereinafter only referred as HumanCyc) [19] or Chemical Entities of Biological Interest (ChEBI) [20].

We analysed the current completeness of the following metabolite libraries: Human Metabolome Database (HMDB) [21], KEGG, PubChem, HumanCyc, ChEBI, ChemSpider [22], the metabolic reconstruction Recon2 [23], METLIN [24] and Lipid Metabolites and Pathways Strategy (LipidMAPS) [25].

The list of significant metabolites from [11-15] was used to assess the completeness of these nine databases. The identification of metabolites had been carried out by original authors in all the datasets, and in some cases KEGG and HMDB identifiers were already provided by authors. Since the HMDB website provides links to other metabolite databases, we started the search of the

Table 1 Main characteristics of the datasets used, extracted from the repository MetabolomeXchange

\begin{tabular}{|c|c|c|c|c|c|c|}
\hline Dataset & $\begin{array}{l}\text { Repository } \\
\text { reference }\end{array}$ & $\begin{array}{l}\text { Condition } \\
\text { of study }\end{array}$ & $\begin{array}{l}\text { Metabolomic } \\
\text { platform }\end{array}$ & $\begin{array}{l}\text { Significant metabolites } \\
\text { in publication }\end{array}$ & $\begin{array}{l}\text { Total metabolites } \\
\text { analysed by authors }\end{array}$ & Reference \\
\hline 1 & ST000091 & Type 1 diabetes & LC(RP)-MS & 8 & 44 & [11] \\
\hline 2 & ST000383 & $\begin{array}{l}\text { Type } 2 \text { diabetes } \\
\text { and obesity }\end{array}$ & GC-MS & 27 & 106 & [12] \\
\hline 3 & MTBLS364 & Smokers & NMR, LC(HILIC-/RP)-MS & 81 & - & [13] \\
\hline 4 & MTBLS424 & Breast cancer & NMR & 22 & 25 & [14] \\
\hline 5 & ST000284 & Colorectal cancer & LC(RP)-MS & 42 & 113 & [15] \\
\hline
\end{tabular}

Abbreviations: GC gas chromatography, HILIC hydrophilic interaction liquid chromatography, LC liquid chromatography, MS mass spectrometry, $N M R$ nuclear magnetic resonance, $R P$ reverse phase 
identifiers on this site and then we extended this search to the LipidMAPS website and MetExplore [26] (for Recon2 codes). All the identifiers were then doublechecked in the corresponding metabolite databases. If more than one metabolite identifier was found (e.g. in PubChem or ChEBI databases), we took all those identifiers and checked in ConsensusPathDB [27] which ones were recognized by the tool (not shown). When the stereochemistry of the metabolite was not specified, the most common chemical configuration was assumed. The complete list of metabolite identifiers is shown in Additional file 1: Table S1.

\section{Generation of enriched data}

Most of the bioinformatic tools for the enrichment of metabolomics datasets accept a list of identifiers as output, while a lower number require quantitative data, e.g. concentration, fold change or peak intensity. Therefore we decided to work with a list of metabolites (name or identifier) from real datasets and enriched data to compare these tools. Although this approach do not allow us to assess some of the available tools, i.e. 3omics or PAPi, the use of simulated o synthetic data would have allowed us to examine a lower number of tools.

For data enrichment, the dataset with the most metabolites was selected (colorectal cancer, ST000284). The list of significant metabolites of this dataset $(n=42$, obtained from [15]) was analysed with MetaboAnalyst [28], using the option 'pathway analysis'. MetaboAnalyst's output was examined and the three KEGG pathways that presented the lowest false discovery rate (FDR), based on the Benjamini-Hochberg procedure [29], were chosen for pathway enrichment: 1) Alanine, aspartate and glutamate metabolism; 2) Aminoacyl-tRNA biosynthesis, and 3) Arginine and proline metabolism.

The R package KEGGREST (v.1.17.0) [30] was employed to build an adjacency matrix [31] which linked the metabolites of the dataset $(n=113)$ with their corresponding KEGG pathways. One was assigned if the metabolite was part of that particular pathway, or 0 if not. Then five metabolites of each pathway were randomly sampled. Enriched data are shown in Additional file 2: Table S2.

\section{Statistical analysis \\ Similarity analysis}

The most commonly used tools for metabolomics data enrichment were chosen for similarity analysis. This selection was formed by 3omics [32], BioCyc/HumanCyc [19], ConsensusPathDB, IMPaLA [33], Ingenuity ${ }^{\oplus}$ Pathway Analysis (IPA ${ }^{\circ}$ QIAGEN, Redwood City, CA), KEGG [16], MassTRIX [34], MBRole [1], MetaboAnalyst, Metabox [35], MetaCore ${ }^{\mathrm{TM}}$ (Thomson Reuters Inc., Carlsbad, CA), MetaMapp [36], MetExplore, MetScape [37], MPEA [38], PaintOmics [39], PAPi [40], PathVisio
[41], Reactome [42], Small Molecule Pathway Database (SMPDB) [43], WikiPathways [44] and XCMS [45].

The main features of these tools were summarized on a binary matrix (Yes/No responses) including whether they 1) perform ORA, integration with other 'omics' or other enrichment analyses; 2) visualization of pathways, networks or other types of visualization; 3) use KEGG, BioCyc, Reactome, Wikipathways, SMPDB or other pathway databases; 4) are databases, programmable, open-source or online tools (Additional file 3: Table S3).

The similarity analysis was performed with the $\mathrm{R}$ package vegan (v.2.4-4) [46]. First, the Yes/No responses were transformed to 1 and 0 , respectively. Then Jaccard's coefficients were calculated and a non-metric multidimensional scaling (NMDS) was performed. This method plots dissimilar objects far apart in the ordination space and similar objects close to one another preserving ordering relationships among them [47].

\section{Over-representation analysis}

The performance of the tools that perform ORA in metabolomics datasets was assessed with the list of significant metabolites of the colorectal cancer dataset (ST000284) $(n=42)$ [15] and of enriched data. The comparative analysis of ORA tools was performed on tools employing KEGG, Reactome and HumanCyc as pathway database. The selected tools were ConsensusPathDB, HumanCyc, IMPaLA, MBRole, MetaboAnalyst, Metabox, MetExplore, MPEA, PathVisio and Reactome and the pathway mapping tool KEGGREST. Table 2 summarizes the main features of these tools and type of identifiers used. Analyses were performed following the guidelines of each tool.

The output of these tools was examined for the following metabolic pathways: 1) KEGG: the three aforementioned pathways; 2) Reactome: Metabolism of amino acids and derivates pathway; and 3) HumanCyc: tRNA charging pathway. Ranking (position in the list of pathways sorted by significance), total number of metabolites/pathway, number of hits/pathway, p- and adjusted $p$-value (generally FDR, calculated by the tools) were recorded from each output.

\section{Disease-based enrichment analyses}

Disease-based enrichment analyses were performed by using the list of significant metabolites of the five datasets on: 1) MetaboAnalyst (SMPDB disease pathway database) [48]; 2) MBRole (HMDB disease database); 3) IPA $^{\oplus}$ (Ingenuity ${ }^{\oplus}$ disease database); and 4) MetaCore $^{{ }^{\mathrm{TM}}}$ ( $\mathrm{MeSH}$ and OMIM disease databases). Disease, ranking (position according to their $p$-value), total number of metabolites/disease, number of hits/disease, $\mathrm{p}$ - and adjusted $p$-values were recorded from each output. 
Table 2 Summary of the tools used to assess the performance of over-representation (ORA) methods and their main characteristics (July 2017). Tools and databases are sorted alphabetically

\begin{tabular}{|c|c|c|c|c|c|c|c|}
\hline $\begin{array}{l}\text { Tool } \\
\text { name }\end{array}$ & $\begin{array}{l}\text { Tool } \\
\text { version }\end{array}$ & $\begin{array}{l}\text { Database } \\
\text { used }\end{array}$ & $\begin{array}{l}\text { Database } \\
\text { version }\end{array}$ & $\begin{array}{l}\text { Test used in } \\
\text { this work }\end{array}$ & Platform & $\begin{array}{l}\text { Input } \\
\text { code }\end{array}$ & Website \\
\hline ConsensusPathDB & 32 & $\begin{array}{l}\text { HumanCyc } \\
\text { KEGG } \\
\text { Reactome }\end{array}$ & $\begin{array}{l}19.1 \\
(06 / 2015) \\
80.0 \\
(10 / 2016) \\
59 \\
(12 / 2016)\end{array}$ & $\begin{array}{l}\text { Fisher's exact test } \\
\text { Fisher's exact test } \\
\text { Fisher's exact test }\end{array}$ & Online & $\begin{array}{l}\text { HumanCyc } \\
\text { KEGG } \\
\text { Reactome }\end{array}$ & http://cpdb.molgen.mpg.de/ \\
\hline HumanCyc & 21.0 & HumanCyc & $\begin{array}{l}21.0 \\
(12 / 2016)\end{array}$ & Fisher's exact test & Online & Name & https://humancyc.org/ \\
\hline IMPaLA & 10 & $\begin{array}{l}\text { HumanCyc } \\
\text { KEGG } \\
\text { Reactome }\end{array}$ & $\begin{array}{l}\text { NA } \\
\text { NA } \\
\text { NA }\end{array}$ & $\begin{array}{l}\text { Fisher's exact test } \\
\text { Fisher's exact test } \\
\text { Fisher's exact test }\end{array}$ & Online & $\begin{array}{l}\text { HumanCyc } \\
\text { KEGG } \\
\text { Reactome }\end{array}$ & http://impala.molgen.mpg.de/ \\
\hline $\mathrm{IPA}^{\oplus}$ & NA & IPA $A^{\oplus}$ disease & NA & $\begin{array}{l}\text { Fisher's exact test, } \\
\text { Z-score }\end{array}$ & $\begin{array}{l}\text { Java-based } \\
\text { software }\end{array}$ & KEGG & \\
\hline KEGGREST & 1.17 .0 & KEGG & NA & - & $\mathrm{R}$ & KEGG & $\begin{array}{l}\text { https://bioconductor.org/packages/release/bioc/ } \\
\text { html/KEGGREST.html }\end{array}$ \\
\hline MBRole & 2.0 & $\begin{array}{l}\text { HMDB } \\
\text { disease } \\
\text { HumanCyc } \\
\text { KEGG }\end{array}$ & $\begin{array}{l}3.5 \\
(01 / 2013) \\
17.1 \\
(06 / 2013) \\
54.1 \\
(05 / 2010)\end{array}$ & $\begin{array}{l}\text { Hypergeometric test } \\
\text { Hypergeometric test } \\
\text { Hypergeometric test }\end{array}$ & Online & $\begin{array}{l}\text { HumanCyc } \\
\text { KEGG }\end{array}$ & http://csbg.cnb.csic.es/mbrole2/ \\
\hline MetaboAnalyst & 3.0 & $\begin{array}{l}\text { SMPDB } \\
\text { disease } \\
\text { KEGG }\end{array}$ & $\begin{array}{l}\text { NA } \\
\text { NA }\end{array}$ & $\begin{array}{l}\text { Fisher's exact test, } \\
\text { hypergeometric test } \\
\text { Fisher's exact test, } \\
\text { hypergeometric test }\end{array}$ & Online & KEGG & http://www.metaboanalyst.ca/ \\
\hline Metabox & NA & KEGG & NA & Hypergeometric test & $\mathrm{R}$ & PubChem & https://github.com/kwanjeeraw/metabox \\
\hline MetaCore $^{T M}$ & NA & $\begin{array}{l}\text { MeSH and } \\
\text { OMIM disease }\end{array}$ & NA & - & Online & PubChem & https://portal.genego.com/ \\
\hline MetExplore & 2.11 .2 & $\begin{array}{l}\text { HumanCyc } \\
\text { KEGG }\end{array}$ & $\begin{array}{l}18.0 \\
(02 / 2014) \\
74.0 \\
(04 / 2015)\end{array}$ & $\begin{array}{l}\text { Fisher's exact test } \\
\text { Fisher's exact test }\end{array}$ & Online & $\begin{array}{l}\text { HumanCyc } \\
\text { KEGG }\end{array}$ & http://metexplore.toulouse.inra.fr/metexplore2/ \\
\hline MPEA & (2010) & KEGG & (2010) & Hypergeometric test & Online & KEGG & http://ekhidna.biocenter.helsinki.fi/poxo/mpea \\
\hline PathVisio & 3.2 .4 & Reactome & $\begin{array}{l}54 \\
(10 / 2015)\end{array}$ & Z-score & $\begin{array}{l}\text { Java-based } \\
\text { software }\end{array}$ & KEGG & https://www.pathvisio.org/ \\
\hline Reactome & 61 & Reactome & $\begin{array}{l}61 \\
(06 / 2017)\end{array}$ & Fisher's exact test & Online & KEGG & http://reactome.org/ \\
\hline
\end{tabular}

Abbreviations: NA not available

\section{Results}

Evaluation of the state-of-the-art of bioinformatic tools

Figure 1 displays a similarity plot of the most commonly used bioinformatic tools. Tools were distributed all along the two dimensions revealing their diversity. The first dimension mainly separated tools that: 1) perform ORA and are non-open source, 2) perform ORA and are opensource, and 3) are a metabolite database. On the other hand, the second dimension mainly separated tools that: 1) perform metabolite identification, 2) perform ORA and are not programmable, and 3) perform ORA and are programmable. MetScape and MetaMapp, which only carry out data visualization, were distant in the plot.

\section{Evaluation of the completeness of metabolite databases} Metabolites of the five datasets were used to assess the completeness of the metabolite and pathway databases.
Almost all the metabolites presented PubChem (97\%), ChEBI (91\%), METLIN (91\%), KEGG (88\%), ChemSpider (87\%) and HMDB (86\%) identifiers, and the $97 \%$ of the lipid subset had LipidMAPS identifiers. In some cases, KEGG, HumanCyc and Recon 2 provided chemical class identifiers instead of a single identifier to certain metabolites, especially to the lipid subset (Additional file 1: Table S1 and Additional file 4: Table S4).

\section{Evaluation of over-representation methods}

In general, ORA methods yielded consistent results using both real and enriched data in all the range of tools tested (Tables 3 and 4). Also similar results were obtained in paired analyses/tools such as MetaboAnalyst hypergeometric test - Fisher's exact test, MBRole full Homo sapiens database, MPEA top down - bottom up 


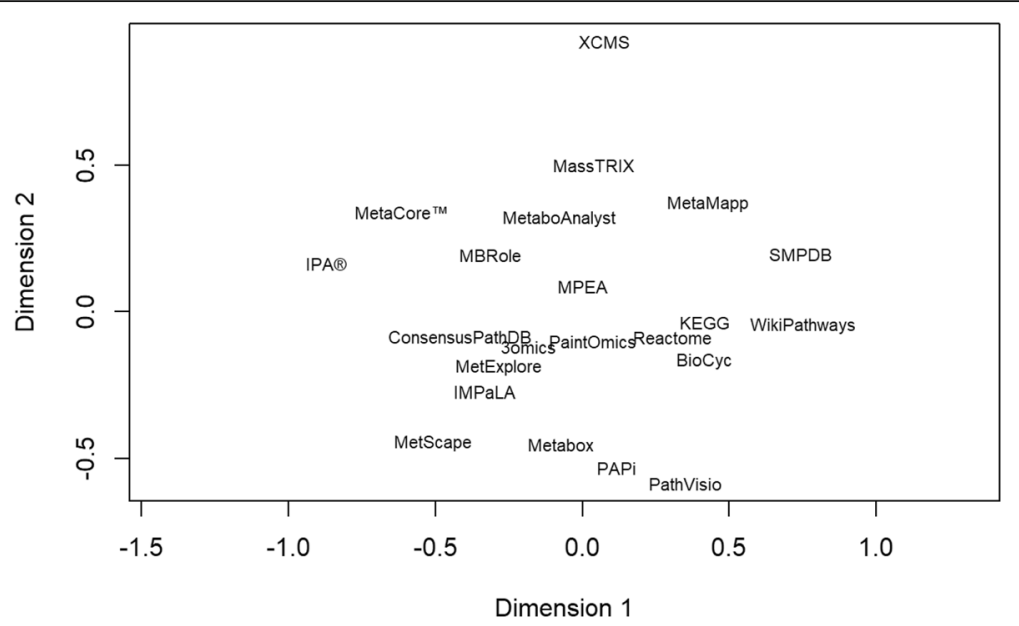

Fig. 1 Non-metric multidimensional scaling (NMDS) plot of the most used tools for metabolomics data enrichment based on Jaccard's distances. Additional file 3: Table S3 shows the main features of each tool

analyses, or ConsensusPathDB - IMPaLA tools, as expected (Tables 3 and 4).

Minor differences in the total number of metabolites/ pathway and number hits/pathway were found. For instance, MPEA (Table 3) and MBRole (Table 4) presented a higher number of metabolites/pathway than the other tools. Other divergences were also observed, e.g. MPEA provided higher adjusted $p$-values values (nearly 1 in all the cases) than other tools, or not all the tools mapped the same metabolites of the dataset onto the queried pathways (not shown).

\section{Evaluation of disease-based libraries}

The significant metabolites of the five datasets were used to analyse the accuracy of the SMPDB, HMDB, IPA ${ }^{\oplus}, \mathrm{MeSH}$ and OMIM disease-based libraries. Outputs revealed that the diseases queried (diabetes type 1 and 2, obesity, respiratory alterations and breast and colorectal cancer) were not successfully identified by these tools, as they appeared in a low position in the list of potential diseases and most of the times they presented a $p>0.05$ (Table 5).

\section{Discussion}

Interpretation of metabolomic data is much less straightforward than that with genomic and proteomic datasets [36]. In the present work we have described the diversity of bioinformatic tools for metabolite sets and have evaluated their performance by exploring three features: the completeness of metabolite databases, ORA approaches and disease-based analyses. To that end, we have used five metabolite sets of blood biomarkers of different diseases obtained from LC-MS, GC-MS and NMR metabolomics approaches. This approach allowed minimizing the possible bias introduced by a given metabolomic platform and thus working with a wide range of metabolites.
Metabolomics is a developing field, thus bioinformatic tools designed to perform enrichment of metabolomics datasets are being developed and released by various groups using diverse statistical tests [3]. Our exploratory multivariate analysis mirrors the high diversity of the currently available tools for the analysis of metabolite sets.

To date about 30,000 endogenous metabolites have already been identified, but this number is rapidly increasing due to advances in high-throughput technologies [21]. Current metabolite databases do not have the full potential to quickly absorb these advances in the description of the endogenous metabolome yet, as not a single metabolite database used in this work covered the full list of significant metabolites of the five datasets. Among all the metabolites databases, PubChem was the one that covered more metabolites from the datasets. However, PubChem is a crowded compound database and presents duplicated metabolite entries, which might produce a larger number of false positives than searching against the KEGG database [49]. To address the low metabolite coverage of metabolite databases, some of them such as KEGG and HumanCyc assign chemical class identifiers to certain types of compounds, especially lipids such as phosphatidylcholines, sphingomyelins or triglycerides. For instance, KEGG coded phosphatidylcholines and sphingomyelins as 'C00157' and 'C00550', respectively, and HumanCyc as 'PHOSPHATIDYLCHOLINE' and 'Sphyngomyelin (class)'.

Missing, ambiguous or redundant entries have been commonly found in public repositories [50]. Indeed metabolites with more than one PubChem, HMDB or ChEBI identifiers were found in this work, which reduce enrichment analyses' accuracy. Several on-going initiatives on identifiers standardization such as BridgeDB and the Chemical Translation Service are trying to 
Table 3 Evaluation of over-representation analysis (ORA) outputs of bioinformatic tools employing KEGG pathways. Real (from dataset ST000284) and enriched data were used. The number of total metabolites in the pathway, the number of hits, the ranking of the pathway among all the KEGG pathways (according to their significance), the $p$-value and the adjusted $p$-value were calculated by the tools

\begin{tabular}{|c|c|c|c|c|c|c|}
\hline Tool & Data & Rank & Total metab. & Hits & $P$-value & Adjusted $p$-value \\
\hline \multicolumn{7}{|c|}{ Alanine, aspartate and glutamate metabolism } \\
\hline \multirow[t]{2}{*}{ ConsensusPathDB } & Real & 2 & 28 & 8 & $3.77 \mathrm{E}-11$ & $9.99 \mathrm{E}-10$ \\
\hline & Enriched & 2 & 28 & 7 & $3.76 \mathrm{E}-13$ & 7.32E-12 \\
\hline \multirow[t]{2}{*}{ IMPaLA } & Real & 2 & 28 & 8 & $3.77 \mathrm{E}-11$ & 7.91E-09 \\
\hline & Enriched & 2 & 28 & 7 & $3.76 \mathrm{E}-13$ & $3.00 \mathrm{E}-10$ \\
\hline \multirow[t]{2}{*}{ KEGGREST } & Real & NA & 28 & 7 & NA & NA \\
\hline & Enriched & NA & 28 & 7 & NA & NA \\
\hline \multirow[t]{2}{*}{ MBRole (full database) } & Real & 2 & 24 & 8 & $3.47 \mathrm{E}-12$ & $2.07 \mathrm{E}-10$ \\
\hline & Enriched & 1 & 24 & 7 & 7.23E-14 & $5.86 \mathrm{E}-12$ \\
\hline \multirow[t]{2}{*}{ MBRole (Homo sapiens) } & Real & 1 & 24 & 8 & $2.31 \mathrm{E}-11$ & $1.50 \mathrm{E}-09$ \\
\hline & Enriched & 1 & 24 & 7 & $3.85 \mathrm{E}-13$ & $2.00 \mathrm{E}-11$ \\
\hline \multirow[t]{2}{*}{ MetaboAnalyst (Fisher) } & Real & 1 & 24 & 7 & $3.91 \mathrm{E}-06$ & $6.74 \mathrm{E}-05$ \\
\hline & Enriched & 1 & 24 & 7 & $6.21 \mathrm{E}-12$ & 4.97E-10 \\
\hline \multirow[t]{2}{*}{ MetaboAnalyst (hyper.) } & Real & 1 & 24 & 7 & $3.91 \mathrm{E}-06$ & $6.74 \mathrm{E}-05$ \\
\hline & Enriched & 1 & 24 & 7 & $6.21 \mathrm{E}-12$ & 4.97E-10 \\
\hline \multirow[t]{2}{*}{ Metabox } & Real & 2 & 32 & 8 & $3.60 \mathrm{E}-11$ & $5.22 \mathrm{E}-10$ \\
\hline & Enriched & 2 & 32 & 7 & $1.34 \mathrm{E}-13$ & $1.27 \mathrm{E}-12$ \\
\hline \multirow[t]{2}{*}{ MetExplore } & Real & 3 & NA & 8 & $1.03 \mathrm{E}-08$ & $4.32 \mathrm{E}-07$ \\
\hline & Enriched & 2 & NA & 7 & $4.42 \mathrm{E}-10$ & $1.33 \mathrm{E}-08$ \\
\hline \multirow[t]{2}{*}{ MPEA (top down analysis) } & Real & 1 & 24 & 8 & $4.41 \mathrm{E}-11$ & 0.660 \\
\hline & Enriched & 1 & 24 & 7 & $5.01 \mathrm{E}-13$ & 0.440 \\
\hline \multirow[t]{2}{*}{ MPEA (bottom up analysis) } & Real & 1 & 24 & 8 & $1.01 \mathrm{E}-11$ & 0.170 \\
\hline & Enriched & 1 & 24 & 7 & $1.08 \mathrm{E}-12$ & 1.00 \\
\hline \multicolumn{7}{|l|}{ Aminoacyl-tRNA biosynthesis } \\
\hline \multirow[t]{2}{*}{ ConsensusPathDB } & Real & 4 & 52 & 8 & $7.89 \mathrm{E}-10$ & $1.05 \mathrm{E}-07$ \\
\hline & Enriched & 9 & 52 & 5 & $2.58 \mathrm{E}-07$ & $1.12 \mathrm{E}-06$ \\
\hline \multirow[t]{2}{*}{ IMPaLA } & Real & 4 & 52 & 8 & 7.89E-09 & $8.74 \mathrm{E}-07$ \\
\hline & Enriched & 9 & 52 & 5 & $2.58 \mathrm{E}-07$ & $1.13 \mathrm{E}-05$ \\
\hline \multirow[t]{2}{*}{ KEGGREST } & Real & NA & 52 & 8 & NA & NA \\
\hline & Enriched & NA & 52 & 5 & NA & NA \\
\hline \multirow[t]{2}{*}{ MBRole (full database) } & Real & 5 & 75 & 8 & $6.07 \mathrm{E}-08$ & $1.20 \mathrm{E}-06$ \\
\hline & Enriched & 12 & 75 & 5 & $1.23 \mathrm{E}-06$ & 8.30E-06 \\
\hline \multirow[t]{2}{*}{ MBRole (Homo sapiens) } & Real & 5 & 75 & 8 & $3.75 \mathrm{E}-07$ & 4.87E-06 \\
\hline & Enriched & 6 & 75 & 5 & $3.95 \mathrm{E}-06$ & $3.42 \mathrm{E}-05$ \\
\hline \multirow[t]{2}{*}{ MetaboAnalyst (Fisher) } & Real & 3 & 75 & 8 & 1.40E-05 & $3.75 \mathrm{E}-04$ \\
\hline & Enriched & 7 & 75 & 5 & $2.72 \mathrm{E}-05$ & $3.11 \mathrm{E}-04$ \\
\hline \multirow[t]{2}{*}{ MetaboAnalyst (hyper.) } & Real & 3 & 75 & 8 & $1.40 \mathrm{E}-05$ & $3.75 \mathrm{E}-04$ \\
\hline & Enriched & 7 & 75 & 5 & $2.72 \mathrm{E}-05$ & $3.11 \mathrm{E}-04$ \\
\hline \multirow[t]{2}{*}{ Metabox } & Real & 4 & 56 & 8 & $4.28 \mathrm{E}-09$ & $3.10 \mathrm{E}-08$ \\
\hline & Enriched & 4 & 56 & 5 & 8.69E-08 & $2.15 \mathrm{E}-07$ \\
\hline \multirow[t]{2}{*}{ MetExplore } & Real & 5 & NA & 8 & $1.55 \mathrm{E}-06$ & $1.69 \mathrm{E}-06$ \\
\hline & Enriched & 7 & NA & 5 & $1.51 \mathrm{E}-05$ & $4.52 \mathrm{E}-04$ \\
\hline
\end{tabular}


Table 3 Evaluation of over-representation analysis (ORA) outputs of bioinformatic tools employing KEGG pathways. Real (from dataset ST000284) and enriched data were used. The number of total metabolites in the pathway, the number of hits, the ranking of the pathway among all the KEGG pathways (according to their significance), the $p$-value and the adjusted $p$-value were calculated by the tools (Continued)

\begin{tabular}{|c|c|c|c|c|c|c|}
\hline Tool & Data & Rank & Total metab. & Hits & $P$-value & Adjusted $p$-value \\
\hline \multirow[t]{2}{*}{ MPEA (top down analysis) } & Real & 3 & 53 & 8 & $5.32 \mathrm{E}-09$ & 1.00 \\
\hline & Enriched & 7 & 53 & 5 & $1.42 \mathrm{E}-06$ & 1.00 \\
\hline \multirow[t]{2}{*}{ MPEA (Bottom up analysis) } & Real & 5 & 53 & 8 & $7.12 \mathrm{E}-08$ & 1.00 \\
\hline & Enriched & 4 & 53 & 5 & $6.57 \mathrm{E}-08$ & 1.00 \\
\hline \multicolumn{7}{|l|}{ Arginine and proline metabolism } \\
\hline \multirow[t]{2}{*}{ ConsensusPathDB } & Real & 9 & 76 & 7 & 2.79E-06 & $1.64 \mathrm{E}-05$ \\
\hline & Enriched & 4 & 76 & 6 & $3.94 \mathrm{E}-08$ & $3.85 \mathrm{E}-07$ \\
\hline \multirow[t]{2}{*}{ IMPaLA } & Real & 9 & 76 & 7 & 2.79E-09 & $8.74 \mathrm{E}-07$ \\
\hline & Enriched & 4 & 76 & 6 & $3.94 \mathrm{E}-08$ & $2.18 \mathrm{E}-06$ \\
\hline \multirow[t]{2}{*}{ KEGGREST } & Real & NA & 77 & 7 & NA & NA \\
\hline & Enriched & NA & 77 & 6 & NA & NA \\
\hline \multirow[t]{2}{*}{ MBRole (full database) } & Real & 3 & 82 & 10 & $2.59 \mathrm{E}-10$ & $8.55 \mathrm{E}-09$ \\
\hline & Enriched & 2 & 82 & 8 & $9.30 \mathrm{E}-12$ & 3.77E-10 \\
\hline \multirow[t]{2}{*}{ MBRole (Homo sapiens) } & Real & 2 & 82 & 10 & $2.58 \mathrm{E}-09$ & $8.38 \mathrm{E}-08$ \\
\hline & Enriched & 2 & 82 & 8 & $6.21 \mathrm{E}-11$ & $1.61 \mathrm{E}-09$ \\
\hline \multirow[t]{2}{*}{ MetaboAnalyst (Fisher) } & Real & 2 & 77 & 9 & $6.69 \mathrm{E}-06$ & $6.74 \mathrm{E}-05$ \\
\hline & Enriched & 2 & 77 & 8 & $8.61 \mathrm{E}-10$ & $3.45 \mathrm{E}-08$ \\
\hline \multirow[t]{2}{*}{ MetaboAnalyst (hyper.) } & Real & 2 & 77 & 9 & $6.69 \mathrm{E}-06$ & $6.74 \mathrm{E}-05$ \\
\hline & Enriched & 2 & 77 & 8 & $8.61 \mathrm{E}-10$ & $3.45 \mathrm{E}-08$ \\
\hline \multirow[t]{2}{*}{ Metabox } & Real & 9 & 84 & 7 & $1.92 \mathrm{E}-05$ & $6.18 \mathrm{E}-05$ \\
\hline & Enriched & 4 & 84 & 6 & $1.25 \mathrm{E}-08$ & 5.96E-08 \\
\hline \multirow[t]{2}{*}{ MetExplore } & Real & 2 & NA & 10 & 4.03E-08 & 1.69E-06 \\
\hline & Enriched & 1 & NA & 8 & $3.34 \mathrm{E}-10$ & $1.00 \mathrm{E}-08$ \\
\hline \multirow[t]{2}{*}{ MPEA (top down analysis) } & Real & 4 & 90 & 10 & $1.40 \mathrm{E}-08$ & 1.00 \\
\hline & Enriched & 2 & 90 & 7 & $1.09 \mathrm{E}-10$ & 1.00 \\
\hline \multirow[t]{2}{*}{ MPEA (bottom up analysis) } & Real & 2 & 90 & 10 & $2.24 \mathrm{E}-09$ & 1.00 \\
\hline & Enriched & 2 & 90 & 8 & 1.69E-10 & 1.00 \\
\hline
\end{tabular}

NA means that information was not provided by the tool. Abbreviations: Fisher Fisher's exact test, hyper hypergeometric test, NA not available

overcome redundancy [50-52]. Some tools such as MetaboAnalyst, ConsensusPathDB or PathVisio embrace these initiatives and accept different types of identifiers, which are then transformed into an internal identifier prior to the enrichment analysis [51]. However, this approach also presents different pitfalls. For instance, these tools usually transform the input code into KEGG identifiers, and thus certain types of metabolites such as lipids lose their uniqueness and become a chemical class KEGG identifier. Consequently bioinformatic tools analyse these lipids as a single entity, thereby losing the diversity of these metabolites.

KEGG and HumanCyc are the most used pathway libraries in metabolomics $[3,17]$ and Reactome is widely used in other 'omics' studies [53]. Thus we have evaluated and compared outputs of ORA methods that employ these pathway libraries. Some limitations prior to ORA analysis were found. For instance, despite the fact that almost all the metabolites of ST000284 dataset had a KEGG code, not all of them were mapped in a KEGG pathway. However, these compounds (e.g. 5-hydroxytryptophan and salicylurate) were mapped in other pathway databases such as Reactome, Wikipathways and SMPDB (not shown). In addition, the KEGG code for glutamic acid (C00025) was not recognized by MetaboAnalyst and the alternative suggested by the tool corresponded to the compound amphetamine (C07514).

The number of total metabolites and hits per pathway varied according to the tool used and those tools that employ the newer database versions (Table 2) presented the higher number of metabolites, as expected. 
Table 4 Evaluation of over-representation analysis (ORA) outputs of bioinformatic tools employing Reactome and HumanCyc pathways. Real (from dataset ST000284) and enriched data were used. The number of total metabolites in the pathway, the number of hits, the ranking of the pathway among all the Reactome or HumanCyc pathways (according to their significance), the $p$-value and the adjusted $p$-value were calculated by the tools

\begin{tabular}{|c|c|c|c|c|c|c|}
\hline Tool & Data & Rank & Total metab. & Hits & $P$-value & Adjusted $p$-value \\
\hline \multicolumn{7}{|l|}{ Reactome } \\
\hline \multicolumn{7}{|c|}{ Metabolism of amino acids and derivates } \\
\hline \multirow[t]{2}{*}{ ConsensusPathDB } & Real & 3 & 272 & 18 & $8.46 \mathrm{E}-14$ & $4.55 \mathrm{E}-12$ \\
\hline & Enriched & 1 & 272 & 12 & 7.67E-15 & $5.75 \mathrm{E}-13$ \\
\hline \multirow[t]{2}{*}{ IMPaLA } & Real & 3 & 272 & 18 & $8.46 \mathrm{E}-14$ & $4.21 \mathrm{E}-11$ \\
\hline & Enriched & 1 & 272 & 12 & 7.67E-15 & $1.02 \mathrm{E}-11$ \\
\hline \multirow[t]{2}{*}{ PathVisio } & Real & NA & NA & NA & NA & NA \\
\hline & Enriched & NA & NA & NA & NA & NA \\
\hline \multirow[t]{2}{*}{ Reactome } & Real & 9 & 283 & 18 & $1.03 \mathrm{E}-04$ & $3.81 \mathrm{E}-03$ \\
\hline & Enriched & 1 & 283 & 12 & $8.18 \mathrm{E}-08$ & $1.00 \mathrm{E}-05$ \\
\hline \multicolumn{7}{|l|}{ HumanCyc } \\
\hline \multicolumn{7}{|l|}{ tRNA charging } \\
\hline \multirow[t]{2}{*}{ ConsensusPathDB } & Real & 2 & 24 & 8 & $9.14 \mathrm{E}-12$ & $1.92 \mathrm{E}-10$ \\
\hline & Enriched & 2 & 24 & 5 & 4.40E-09 & $1.30 \mathrm{E}-07$ \\
\hline \multirow[t]{2}{*}{ HumanCyc } & Real & 8 & 24 & 8 & 2.57E-05 & 0.002 \\
\hline & Enriched & 18 & 24 & 5 & 7.90E-05 & $4.25 \mathrm{E}-03$ \\
\hline \multirow[t]{2}{*}{ IMPaLA } & Real & 2 & 24 & 8 & $9.14 \mathrm{E}-12$ & $2.28 \mathrm{E}-09$ \\
\hline & Enriched & 2 & 24 & 5 & 4.40E-09 & $3.51 \mathrm{E}-07$ \\
\hline \multirow[t]{2}{*}{ MBRole (full database) } & Real & 4 & 64 & 8 & 8.38E-09 & $1.14 \mathrm{E}-06$ \\
\hline & Enriched & 47 & 64 & 4 & $3.58 \mathrm{E}-07$ & 7.97E-06 \\
\hline \multirow[t]{2}{*}{ MBRole (Homo sapiens) } & Real & 5 & 64 & 8 & $1.26 \mathrm{E}-04$ & $2.44 \mathrm{E}-03$ \\
\hline & Enriched & 11 & 64 & 5 & $1.58 \mathrm{E}-04$ & $1.52 \mathrm{E}-03$ \\
\hline \multirow[t]{2}{*}{ MetExplore } & Real & 1 & NA & 8 & 7.11E-07 & $7.75 \mathrm{E}-05$ \\
\hline & Enriched & 6 & NA & 5 & 4.45E-05 & 3.60E-04 \\
\hline
\end{tabular}

NA means that information was not provided by the tool. Abbreviations: NA not available

Surprisingly, KEGGREST, a R package that provides an updated client interface to the KEGGREST server, did not provide the highest number of total metabolites among the tested KEGG pathways. Despite regular updates to some pathway databases, such as KEGG [16] or Reactome [42], being carried out, most of the tools evaluated do not use up-to-date database versions (Table 2) [54]. Wadi et al. performed an elegant review on the impact of outdated annotations on pathway enrichment analysis, which revealed that many software tools use functional information not updated for years, thereby strongly affecting the quality of the analyses [54].

We can conclude that current ORA methods, despite their differences, provide consistent, robust and reproducible results regardless of their analytic approach (statistical test, $p$-value adjustment or pathway database used), despite the limitations and small differences found between outputs. The most discordant result was obtained with MPEA, probably due to the fact that it employs a different method to handle many-to-many relationships that may occur between the query compounds and metabolite annotations [38].

Although we cannot recommend one tool over the others, we suggest choosing those tools that employ updated metabolite/pathway databases in order to obtain more complete results. Nevertheless, we also consider that the enrichment analysis must not be restricted to a single database or tool. The combined use of libraries such as KEGG, Reactome, HumanCyc or WikiPathways will increase the metabolome coverage and the statistical power of the enrichment analysis.

Disease-based enrichment analysis did not yield accurate results. Although we only used serum/plasma biomarkers, results with other types of biological samples would have been similar. On one hand, metabolite disease sets are not up-to-date. For instance, MetaboAnalyst and MBRole (SMPDB and HMDB disease databases, respectively) base their searches of literature dated 
Table 5 Disease-based enrichment analyses of the five datasets performed with MetaboAnalyst (SMPDB disease database), MBRole (HMDB disease database) and IPA ${ }^{\circledast}$ (in-house disease database) and MetaCore (based on MeSH and OMIM annotations). When the exact disease/condition of study was not obtained, a similar disease was selected

\begin{tabular}{|c|c|c|c|c|c|c|c|}
\hline Dataset & Disease input & Disease output & Rank & Input number metabolites & Hits output & $P$-value & Adjusted $p$-value \\
\hline \multicolumn{8}{|l|}{ MetaboAnalyst } \\
\hline ST000091 & Type 1 diabetes mellitus & Diabetes mellitus MODY & 20 & 8 & 2 & $3.40 \mathrm{E}-02$ & $5.84 \mathrm{E}-01$ \\
\hline \multirow[t]{2}{*}{ ST000383 } & Type 2 diabetes mellitus & Diabetes mellitus MODY & 4 & 27 & 4 & 8.60E-03 & $6.69 \mathrm{E}-01$ \\
\hline & Obesity & Obesity & 31 & 27 & 1 & 9.07E-02 & 8.83E-01 \\
\hline MTBLS364 & Smokers & - & - & 81 & - & - & - \\
\hline MTBLS424 & Breast cancer & Mammary tumour & 30 & 22 & 2 & 4.08E-03 & 4.68E-02 \\
\hline ST000284 & Colorectal cancer & Cervical/colon/ovarian cancer & 46 & 42 & 1 & 8.47E-02 & 5.30E-01 \\
\hline \multicolumn{8}{|l|}{ MBRole } \\
\hline ST000091 & Type 1 diabetes mellitus & - & - & 8 & - & - & - \\
\hline \multirow[t]{2}{*}{ ST000383 } & Type 2 diabetes mellitus & Type 2 diabetes mellitus & 8 & 27 & 3 & $1.16 \mathrm{E}-02$ & $5.48 \mathrm{E}-02$ \\
\hline & Obesity & Obesity & 28 & 27 & 1 & $1.08 \mathrm{E}-01$ & $1.48 \mathrm{E}-01$ \\
\hline MTBLS364 & Smokers & Lung Cancer & 16 & 81 & 31 & $3.02 \mathrm{E}-02$ & $9.25 \mathrm{E}-02$ \\
\hline MTBLS424 & Breast cancer & Lung Cancer & 7 & 22 & 6 & $1.27 \mathrm{E}-04$ & 1.09E-03 \\
\hline ST000284 & Colorectal cancer & Colorectal cancer & 44 & 42 & 1 & 5.19E-02 & 1.14E-01 \\
\hline \multicolumn{8}{|l|}{$\mathrm{IPA}^{\oplus}$} \\
\hline ST000091 & Type 1 diabetes mellitus & - & - & 8 & - & - & - \\
\hline \multirow[t]{2}{*}{ ST000383 } & Type 2 diabetes mellitus & Insulin resistance & 21 & 27 & 3 & $6.10 \mathrm{E}-05$ & NA \\
\hline & Obesity & Adipogenesis of fat & 264 & 27 & 1 & $1.54 \mathrm{E}-02$ & NA \\
\hline MTBLS364 & Smokers & Cough & 490 & 81 & 11 & 4.33E-02 & NA \\
\hline MTBLS424 & Breast cancer & Gastric cancer & 2 & 22 & 9 & 5.03E-11 & NA \\
\hline ST000284 & Colorectal cancer & Colorectal cancer & 3 & 42 & 11 & 2.31E-08 & NA \\
\hline \multicolumn{8}{|l|}{ MetaCore ${ }^{\mathrm{TM}}$} \\
\hline ST000091 & Type 1 diabetes mellitus & Type 1 diabetes mellitus & NA & 8 & 0 & NA & NA \\
\hline \multirow[t]{2}{*}{ ST000383 } & Type 2 diabetes mellitus & Type 2 diabetes mellitus & NA & 27 & 7 & NA & NA \\
\hline & Obesity & Obesity & NA & 27 & 1 & NA & NA \\
\hline MTBLS364 & Smokers & Respiratory disorders & NA & 81 & 1 & NA & NA \\
\hline MTBLS424 & Breast cancer & Breast neoplasms & NA & 22 & 0 & NA & NA \\
\hline ST000284 & Colorectal cancer & Colorectal neoplasms & NA & 42 & 13 & NA & NA \\
\hline
\end{tabular}

Abbreviations: NA not available

between 1975 and 2008, as stated in the outputs of these tools. Since 2008, advances in high-throughput techniques have remarkably improved metabolomics analyses and, consequently, more knowledge about these diseases is available. As previously discussed, the use of not updated annotation sets strongly affect the quality of the analyses [54]. On the other hand, metabolites can overlap between unrelated physiopathological events since similar metabolic processes are altered [55]. This fact could complicate the development and accuracy of background sets for disease-based enrichment analysis.

Although extensive work in developing bioinformatic tools for metabolite sets has been carried out in recent years, more effort in improving metabolite/pathway databases and tools is still needed. On one hand, metabolite databases have to rapidly absorb new information from unstoppable advances in high-throughput technologies. On the other hand, enrichment methods should include a wider range of metabolite identifiers (e.g. LipidMAPS, ChemSpider or METLIN) and metabolite pathway databases in order to increase the metabolome coverage. For instance, the LipidMAPS Structure Database contains about 30,000 human endogenous lipids and 12,000 plant lipids, but also databases based on lipid metabolism and signalling pathways, MS/MS spectra and protein-related data [25, 56]. ChemSpider is a general chemical database and offers access to information for almost 25 million experimentally determined structures of natural and synthetic compounds [22]. However, similarly to PubChem, ChemSpider may lead 
to a high number of false positives [57]. The METLIN database includes nearly 1,000,000 molecules, ranging from lipids, steroids, plant \& bacteria metabolites, small peptides, carbohydrates, exogenous drugs/metabolites and central carbon metabolites, and more than 200,000 MS/MS spectra [24]. Including these information sources in current bioinformatic tools would also involve more effort in the improvement of metabolite identifiers converters. Therefore, there is still a long way ahead to achieve complete metabolite and pathway databases and thus accurate enrichment analyses of metabolite sets.

\section{Conclusions}

We have extensively reviewed, for the first time, the state-of-the-art of bioinformatic tools for the enrichment of metabolite sets from metabolomics studies, visualized their diversity, and examined their performance. The redundancy of identifiers, the use of chemical class identifiers and the incompleteness of metabolite databases and disease metabolite sets limit the extent of the analyses and reduce their accuracy. In general, ORA tools provided consistent results among tools revealing that these analyses are robust and reproducible regardless of their analytic approach. However, more work in the completeness of metabolite/pathway databases is required to get more accurate and global insights of the metabolome.

\section{Additional files}

Additional file 1: Table S1. Full list of significant metabolites of the five datasets used in the present study (adjusted $P$-value $<0.05$ ). Dataset A refers to dataset ST000091, B to ST000383, C to MTBLS364, D to MTBLS424 and E to ST000284. (XLSX $28 \mathrm{~kb}$ )

Additional file 2: Table S2. Enriched data and their main metabolite identifiers for ORA analysis. (XLSX $9 \mathrm{~kb}$ )

Additional file 3: Table S3. List of features of the tools analysed by multiple correspondence analysis. Abbreviations: N, no; $Y$, yes. (XLSX $12 \mathrm{~kb}$ )

Additional file 4: Table S4. Number of metabolites with identifiers of the following metabolite databases. Metabolite databases are sorted by the number of identifiers found. *LipidMAPS identifiers were only searched in lipids $(n=67)$, while the rest of identifiers were considered in all the metabolites of the datasets $(n=147)$. (DOCX $16 \mathrm{~kb}$ )

\section{Abbreviations}

ChEBI: Chemical entities of biological interest; FDR: False discovery rate; HMDB: Human metabolome database; KEGG: Kyoto Encyclopaedia of genes and genomes; LipidMAPS: Lipid metabolites and pathways strategy; NMDS: Non-metric multidimensional scaling; ORA: Over-representation analysis; SMPDB: Small molecule pathway database

\section{Acknowledgements}

The authors thank Nina Görner and Claire Lemmens (former members of the University of Barcelona) for their previous work on data visualization.

\section{Funding}

This research was supported by Project PI13/01172 (Plan N de I+ D + i 20132016), co-funded by ISCII-Subdirección General de Evaluación y Fomento de la Investigación; MTM2015/64465-C2-1-R, co-funded by MINECO, and CIBERfes, co-funded by Fondo Europeo de Desarrollo Regional (FEDER). 2014SGR1566 and 2014SGR464 awards from Generalitat de Catalunya's
Agency (AGAUR). AMR and ST acknowledge the Juan de la Cierva postdoctoral fellowship (MINECO), MPR the APIF predoctoral fellowship (University of Barcelona) and MUS the Ramon y Cajal postdoctoral fellowship (MINECO).

Availability of data and materials

All data generated or analysed during the current study are included in this published article and its supplementary information files. Authors state that data are available for further studies.

\section{Declarations}

This manuscript has not been published elsewhere previously and is not being considered by another publication.

All the authors are aware and agree to the content of the paper and their being listed as authors of the manuscript.

\section{Authors' contributions}

The authors' contributions to the manuscript were as follows: AMR, ASP and CAL designed the experiments; AMR and MPR selected the bioinformatic tools; ST and MUS tools provided the metabolite datasets; AB enriched the data and contributed to the statistical analyses; AMR and MPR conducted the analysis of the data; AMR wrote the manuscript; AMR, ASP and CAL contributed to the discussion of the manuscript; all the authors reviewed the manuscript. CAL has the primary responsibility for the final content. All the authors read and approved the final manuscript.

Ethics approval and consent to participate

Not applicable

\section{Competing interests}

The authors declare that they have no competing interests.

\section{Publisher's Note}

Springer Nature remains neutral with regard to jurisdictional claims in published maps and institutional affiliations.

\section{Author details}

${ }^{1}$ Biomarkers \& Nutrimetabolomics Laboratory, Nutrition, Food Science and Gastronomy Department, Food Technology Reference Net (XaRTA), Nutrition and Food Safety Research Institute (INSA-UB), Faculty of Pharmacy and Food Sciences, Pharmacy and Food Science Faculty, University of Barcelona, Barcelona, Spain. ${ }^{2}$ CIBER Fragilidad y Envejecimiento Saludable [CIBERfes], Instituto de Salud Carlos III [ISCIII], Madrid, Spain. ${ }^{3}$ Genetics, Microbiology and Statistics Department, Biology Faculty, University of Barcelona, Barcelona, Spain. ${ }^{4}$ Statistics and Bioinformatics Unit, Vall d'Hebron Institut de Recerca (VHIR), Barcelona, Spain.

Received: 23 August 2017 Accepted: 18 December 2017

Published online: 02 January 2018

\section{References}

1. Chagoyen M, Pazos F. MBRole: enrichment analysis of metabolomic data. Bioinformatics. 2011:27:730-1.

2. Gehlenborg N, O'Donoghue SI, Baliga NS, Goesmann A, Hibbs MA, Kitano H, et al. Visualization of omics data for systems biology. Nat Methods. 2010;7:S56-68.

3. Booth SC, Weljie AM, Turner RJ. Computational tools for the secondary analysis of metabolomics experiments. Comput Struct Biotechnol J. 2013;4:e201301003.

4. Mutation Consequences and Pathway Analysis working group of the International Cancer Genome Consortium. Pathway and network analysis of cancer genomes. Nat Methods. 2015;12:615-21.

5. Misra BB, van der Hooft JJJ. Updates in metabolomics tools and resources: 2014-2015. Electrophoresis. 2016;37:86-110.

6. Khatri P, Sirota M, Butte AJ. Ten years of pathway analysis: current approaches and outstanding challenges. PLoS Comput Biol. 2012;8:e1002375.

7. Khatri P, Draghici S. Ontological analysis of gene expression data: current tools, limitations, and open problems. Bioinformatics. 2005;21:3587-95.

8. Haug K, Salek RM, Steinbeck C. Global open data management in metabolomics. Curr Opin Chem Biol. Elsevier. 2017;36:58-63.

9. Kale NS, Haug K, Conesa P, Jayseelan K, Moreno P, Rocca-Serra P, et al. MetaboLights: an open-access database repository for Metabolomics data. Curr. Protoc. Bioinforma. Hoboken: John Wiley \& Sons, Inc.; 2016. p. 14.13.114.13.18. 
10. Sud M, Fahy E, Cotter D, Azam K, Vadivelu I, Burant C, et al. Metabolomics workbench: an international repository for metabolomics data and metadata, metabolite standards, protocols, tutorials and training, and analysis tools. Nucleic Acids Res. Oxford University Press. 2016:44:D463-70.

11. Lanza IR, Zhang S, Ward LE, Karakelides H, Raftery D, Nair KS. Quantitative Metabolomics by $1 \mathrm{H}-\mathrm{NMR}$ and LC-MS/MS confirms altered metabolic pathways in diabetes. Wang Y, editor. PLoS One. 2010;5:e10538.

12. Fiehn O, Garvey WT, Newman JW, Lok KH, Hoppel CL, Adams SH. Plasma Metabolomic profiles reflective of glucose homeostasis in non-diabetic and type 2 diabetic obese African-American women. Gimble JM, editor. PLoS One. 2010;5:e15234.

13. Kaluarachchi MR, Boulangé CL, Garcia-Perez I, Lindon JC, Minet EF. Multiplatform serum metabolic phenotyping combined with pathway mapping to identify biochemical differences in smokers. Bioanalysis. 2016;8: 2023-43.

14. Hart CD, Vignoli A, Tenori L, Uy GL, Van To T, Adebamowo C, et al. Serum Metabolomic profiles identify ER-positive early breast cancer patients at increased risk of disease recurrence in a multicenter population. Clin Cancer Res. 2017;23:1422-31.

15. Zhu J, Djukovic D, Deng L, Gu H, Himmati F, Chiorean EG, et al. Colorectal cancer detection using targeted serum metabolic profiling. J Proteome Res. 2014;13:4120-30

16. Kanehisa M, Goto S. KEGG: Kyoto encyclopedia of genes and genomes. Nucleic Acids Res. Oxford University Press. 2000;28:27-30.

17. Sas KM, Karnovsky A, Michailidis G, Pennathur S. Metabolomics and diabetes: analytical and computational approaches. Diabetes. 2015;64:718-32.

18. Kim S, Thiessen PA, Bolton EE, Chen J, Fu G, Gindulyte A, et al. PubChem substance and compound databases. Nucleic Acids Res. Oxford University Press. 2016;44:D1202-13.

19. Romero P, Wagg J, Green ML, Kaiser D, Krummenacker M, Karp PD. Computational prediction of human metabolic pathways from the complete human genome. Genome Biol. 2005;6:R2.

20. Degtyarenko K, de Matos P, Ennis M, Hastings J, Zbinden M, McNaught A, et al. ChEBI: a database and ontology for chemical entities of biological interest. Nucleic Acids Res. 2007;36:D344-50.

21. Wishart DS, Jewison T, Guo AC, Wilson M, Knox C, Liu Y, et al. HMDB 3.0-the human Metabolome database in 2013. Nucleic Acids Res. 2013;41:D801-7.

22. Pence $H E$, Williams $A$. ChemSpider: an online chemical information resource. J Chem Educ. American Chemical Society and Division of Chemical Education, Inc. 2010;87:1123-4.

23. Thiele I, Swainston N, Fleming RMT, Hoppe A, Sahoo S, Aurich MK, et al. A community-driven global reconstruction of human metabolism. Nat Biotechnol. 2013;31:419-25.

24. Smith CA, O'Maille G, Want EJ, Qin C, Trauger SA, Brandon TR, et al. METLIN: a metabolite mass spectral database. Ther Drug Monit. 2005;27:747-51.

25. Sud M, Fahy E, Cotter D, Brown A, Dennis EA, Glass CK, et al. LMSD: LIPID MAPS structure database. Nucleic Acids Res. 2007:35:D527-32.

26. Cottret L, Wildridge D, Vinson F, Barrett MP, Charles H, Sagot M-F, et al. MetExplore: a web server to link metabolomic experiments and genomescale metabolic networks. Nucleic Acids Res. 2010;38:W132-7.

27. Kamburov A, Wierling C, Lehrach H, Herwig R. ConsensusPathDB-a database for integrating human functional interaction networks. Nucleic Acids Res. 2009:37:D623-8.

28. Xia J, Wishart DS. Web-based inference of biological patterns, functions and pathways from metabolomic data using MetaboAnalyst. Nat Protoc. 2011;6: 743-60

29. Benjamini Y, Hochberg Y. Controlling the false discovery rate: a practical and powerful approach to multiple testing. J R Stat Soc Ser B. WileyRoyal Statistical Society. 1995;57:289-300.

30. Tenenbaum D. KEGGREST: client-side REST access to KEGG. R Packag. Version. 2013

31. Harary F, Norman RZ, Cartright D. Structural models: an introduction to the theory of directed graphs. New York: John Wiley \& Sons; 1965. p. 415.

32. Kuo T-C, Tian T-F, Tseng Y. 30mics: a web-based systems biology tool for analysis, integration and visualization of human transcriptomic, proteomic and metabolomic data. BMC Syst Biol. 2013;7:64

33. Kamburov A, Cavill R, Ebbels TMD, Herwig R, Keun HC. Integrated pathwaylevel analysis of transcriptomics and metabolomics data with IMPaLA. Bioinformatics. Oxford University Press. 2011;27:2917-8.

34. Suhre K, Schmitt-Kopplin P. MassTRIX: mass translator into pathways. Nucleic Acids Res. 2008;36:W481-4.
35. Wanichthanarak K, Fan S, Grapov D, Barupal DK, Fiehn O. Metabox: a toolbox for Metabolomic data analysis, interpretation and integrative exploration. PLoS One. Public Library of Science. 2017;12:e0171046.

36. Barupal DK, Haldiya PK, Wohlgemuth G, Kind T, Kothari SL, Pinkerton KE, et al. MetaMapp: mapping and visualizing metabolomic data by integrating information from biochemical pathways and chemical and mass spectral similarity. BMC Bioinformatics. 2012;13:99.

37. Gao J, Tarcea VG, Karnovsky A, Mirel BR, Weymouth TE, Beecher CW, et al. Metscape: a Cytoscape plug-in for visualizing and interpreting metabolomic data in the context of human metabolic networks. Bioinformatics. Oxford University Press. 2010;26:971-3.

38. Kankainen M, Gopalacharyulu P, Holm L, Oresic M. MPEA-metabolite pathway enrichment analysis. Bioinformatics. 2011;27:1878-9.

39. García-Alcalde F, García-López F, Dopazo J, Conesa A. Paintomics: a web based tool for the joint visualization of transcriptomics and metabolomics data. Bioinformatics. Oxford University Press. 2011;27:137-9.

40. Aggio RBM, Ruggiero K, Villas-Bôas SG. Pathway activity profiling (PAPi): from the metabolite profile to the metabolic pathway activity. Bioinformatics. 2010;26:2969-76.

41. van lersel MP, Kelder T, Pico AR, Hanspers K, Coort S, Conklin BR, et al. Presenting and exploring biological pathways with PathVisio. BMC Bioinformatics. 2008:9:399.

42. Haw R, Hermjakob H, D'Eustachio P, Stein L. Reactome pathway analysis to enrich biological discovery in proteomics data sets. Proteomics. 2011;11: 3598-613.

43. Frolkis $A$, Knox C, Lim E, Jewison $T$, Law V, Hau DD, et al. SMPDB: the small molecule pathway database. Nucleic Acids Res. 2010;38:D480-7.

44. Pico AR, Kelder T, van lersel MP, Hanspers K, Conklin BR, Evelo C. WikiPathways: pathway editing for the people. PLoS Biol. 2008;6:e184.

45. Smith CA, Want EJ, O'Maille G, Abagyan R, Siuzdak G. XCMS: processing mass spectrometry data for metabolite profiling using nonlinear peak alignment, matching, and identification. Anal Chem. 2006;78:779-87.

46. Oksanen J, Kindt R, Legendre P, O'hara B, Henry M, Maintainer HS. The vegan Package. 2007. http://r-forge.r-project.org/projects/vegan/. Accessed 17 Nov 2017.

47. Legendre P, Legendre L. Numerical ecology. Oxford: Elsevier; 2012. p. 1006

48. Xia J, Wishart DS. MSEA: a web-based tool to identify biologically meaningful patterns in quantitative metabolomic data. Nucleic Acids Res. 2010;38:W71-7.

49. Matsuda F, Shinbo Y, Oikawa A, Hirai MY, Fiehn O, Kanaya S, et al. Assessment of Metabolome annotation quality: a method for evaluating the false discovery rate of elemental composition searches. El-Shemy HA, editor. PLoS One. 2009:4:e7490.

50. Redestig H, Kusano M, Fukushima A, Matsuda F, Saito K, Arita M. Consolidating metabolite identifiers to enable contextual and multiplatform metabolomics data analysis. BMC Bioinformatics. 2010;11:214

51. van lersel MP, Pico AR, Kelder T, Gao J, Ho I, Hanspers K, et al. The BridgeDb framework: standardized access to gene, protein and metabolite identifier mapping services. BMC Bioinformatics. 2010;11:5.

52. Wohlgemuth G, Haldiya PK, Willighagen E, Kind T, Fiehn O. The chemical translation service-a web-based tool to improve standardization of metabolomic reports. Bioinformatics. 2010;26:2647-8.

53. Villaveces JM, Koti P, Habermann BH. Tools for visualization and analysis of molecular networks, pathways, and -omics data. Adv Appl Bioinform Chem. Dove Press. 2015;8:11-22.

54. Wadi L, Meyer M, Weiser J, Stein LD, Reimand J. Impact of outdated gene annotations on pathway enrichment analysis. Nat Methods. 2016;13:705-6.

55. Lindahl A, Forshed J, Nordström A. Overlap in serum metabolic profiles between non-related diseases: implications for LC-MS metabolomics biomarker discovery. Biochem Biophys Res Commun. 2016;478:1472-7.

56. Byrnes RW, Cotter D, Maer A, Li J, Nadeau D, Subramaniam S, et al. An editor for pathway drawing and data visualization in the biopathways workbench. BMC Syst Biol. 2009;3:99.

57. Matsuda F. Rethinking mass spectrometry-based small molecule identification strategies in Metabolomics. Mass Spectrom. (Tokyo, Japan). Mass Spectrometry Society of Japan. 2014;3:S0038. 